\title{
Thermal Radiation, Heat Source/Sink and Work Done by Deformation Impacts on MHD Viscoelastic Fluid over a Nonlinear Stretching Sheet
}

\author{
F. M. Hady ${ }^{1}$, R. A. Mohamed ${ }^{2}$, Hillal M. ElShehabey ${ }^{2,3^{*}}$ \\ ${ }^{1}$ Mathematics Department, Faculty of Science, Assiut University, Assiut, Egypt \\ ${ }^{2}$ Mathematics Department, Faculty of Science, South Valley University, Qena, Egypt \\ ${ }^{3}$ Institute of Mathematics and Scientific Computing, University of Graz, Graz, Austria \\ Email: happliedmath@yahoo.com
}

Received December 16, 2012; revised February 16, 2013; accepted February 23, 2013

Copyright (C) 2013 F. M. Hady et al. This is an open access article distributed under the Creative Commons Attribution License, which permits unrestricted use, distribution, and reproduction in any medium, provided the original work is properly cited.

\begin{abstract}
This work is focused on the effects of heat source/sink, viscous dissipation, radiation and work done by deformation on flow and heat transfer of a viscoelastic fluid over a nonlinear stretching sheet. The similarity transformations have been used to convert the governing partial differential equations into a set of nonlinear ordinary differential equations. These equations are then solved numerically using a very efficient implicit finite difference method. Favorable comparison with previously published work is performed and it is found to be in excellent agreement. The results of this parametric study are shown in several plots and tables and the physical aspects of the problem are highlighted and discussed.
\end{abstract}

Keywords: Flow and Heat Transfer; Second Grade Fluid; Nonlinear Stretching Sheet; Heat Source; Radiation

\section{Introduction}

The study of fluids is different to that of solids as there are differences in physical structure of fluids and solids. The nature of fluids and the way of study are two aspects of fluid mechanics which make it different to solid mechanics. Furthermore, the fluids are categorized to Newtonian and non-Newtonian fluids. The fluids of low molecular weight fall into the Newtonian class and are completely characterized by the Navier-Stokes theory. There is a large variety of materials such as geological materials, liquid foams, polymeric liquids and food products etc. which are capable of flowing but which exhibit flow characteristic that cannot be adequately described by the Navier-Stokes theory. This inadequacy of the NavierStokes theory has led to the development of several theories of non-Newtonian fluids.

Unlike Navier-Stokes fluids, there is not a single model which can completely describe all the properties of the non-Newtonian fluids. They cannot be described in a single model as for Newtonian fluids and there has been much confusion over the classification of non-Newtonian fluids. There are many models describing the properties but not all of non-Newtonian fluids. These models or

${ }^{*}$ Corresponding author. constitutive equations, however, cannot describe all the behaviors of non-Newtonian fluids, e.g., the normal stress relaxation, the elastic effects, and the memory effects. The constitutive equations describing the behaviors of non-Newtonian fluids are more complicated and non linear than those of Newtonian fluids.

As non-Newtonian fluid model Rivlin-Ericksen fluids gained much acceptance from both theorists and experimenters. The special cases of the model, which is the fluid of second grade, are extensively used and a lot of works have been done on the subject. These investigations have been for non-Newtonian fluids of the differential type [1]. In the case of fluids of differential type, the equations of motion are an order higher than the NavierStokes equations, and thus the adherence boundary condition is insufficient to determine the solution completely [2-4] for a detailed discussion of the relevant issues. The same is also true for the approximate boundary layer approximations of the equations of motion. In the absence of a clear means obtaining additional boundary conditions, Beard and Walters [5], in their study of an incompressible fluid of second grade, suggested a method for overcoming this difficulty. They suggested a perturbation approach in which the velocity and the pressure fields were expanded in a series in terms of a small parame- 
ter $\varepsilon$. Danberg and Fansler [6] studied the solution for the boundary layer flow past a wall that is stretched with a speed proportional to the distance along the wall. Rajagopal et al. [7] independently examined the same flow as in [5] and obtained similarity solutions of the boundary layer equations numerically for the case of small viscoelastic parameter $\lambda$. It is shown that skin-friction decreases with increase in $\lambda$. Dandapat and Gupta [8] examined the same problem with heat transfer, where Hady and Gorla [9] studied the effect of uniform suction or injection on flow and heat transfer from a continuous surface in a parallel free stream of viscoelastic secondorder fluid. The effect of radiation on viscoelastic boundary-layer flow and heat transfer problems can be quite significant at high operating temperature. Very recently, researches in these fields have been conducted by many investigators [10-14].

On the other hand, another physical phenomenon is the case in which the sheet stretched in a nonlinear fashion. On this domain, Mahdy and Elshehabey [15] studied the flow and heat transfer in a viscous fluid over a nonlinear stretching sheet utilizing nanofluid where, effects of viscous dissipation and radiation on the thermal boundary layer over a nonlinearly stretching sheet were studied by Cortell [16]. Vajravelu [17] studied viscous flow over a nonlinearly stretching sheet, where viscous flow and heat transfer over a nonlinearly stretching sheet were obtained by Cortell [18] then, series solution of flow over nonlinearly stretching sheet with chemical reaction and magnetic field was investigated by employing the Adomian decomposition method by Kechil and Hashim [19] where, Ziabakhsh et al. [20] used homotopy analysis method to present flow and diffusion of chemically reactive species over a nonlinearly stretching sheet immersed in a porous medium. Muhaimin et al. [21] studied the effect of chemical reaction, heat and mass transfer on nonlinear boundary layer past a porous shrinking sheet in the presence of suction and, Robert [22] discussed high-order nonlinear boundary value problems admitting multiple exact solutions with application to the fluid flow over a sheet. Cortell [23] studied heat and fluid flow due to non-linearly stretching surfaces where, existence and uniqueness results for a nonlinear differential equation arising in viscous flow over a nonlinearly stretching sheet were obtained by Robert et al. [24]. Finally, Vajravelu et al. [25] studied the diffusion of a chemically reactive species of a power-law fluid past a stretching surface.

In this paper, as motivated by the previous studies and the study of Cortell [26] which investigated the effects of heat source/sink, radiation and work done by deformation on flow and heat transfer of a viscoelastic fluid over a stretching sheet, we consider viscoelastic fluid with another physical phenomenon in which the sheet stretched in a nonlinear fashion. Also, the effects of work due to deformation on viscoelastic flows and heat transfer in the presence of radiation, viscous dissipation and heat source/ sink have been studied.

\section{Problem Formulation}

Consider a steady two-dimensional flow of an incompressible second grade fluid through a porous medium over a wall coinciding with the plane $y=0$, the flow being confined to $y>0$. Two equal and opposite forces are applied along the $x$-axis so that the wall is stretched keeping the origin fixed. Thus, the basic boundary layer equations, governing the flow and heat transfer in presence of radiation, with a temperature-dependent heat source/sink in the flow region, viscous dissipation, and taking into account the work due to deformation, are given in usual notation by

$$
\begin{aligned}
& \frac{\partial u}{\partial x}+\frac{\partial v}{\partial y}=0 \\
& u \frac{\partial u}{\partial x}+v \frac{\partial u}{\partial y}=v \frac{\partial^{2} u}{\partial y^{2}}-\frac{\sigma_{0} B_{0}^{2}}{\rho} u \\
& +\frac{\alpha_{1}}{\rho}\left[\frac{\partial}{\partial x}\left(u \frac{\partial^{2} u}{\partial y^{2}}\right)-\frac{\partial u}{\partial y} \frac{\partial^{2} u}{\partial x \partial y}+v \frac{\partial^{3} u}{\partial y^{3}}\right], \\
& u \frac{\partial T}{\partial x}+v \frac{\partial T}{\partial y} \\
& =\alpha \frac{\partial^{2} T}{\partial y^{2}}+\frac{v}{c_{p}}\left(\frac{\partial u}{\partial y}\right)^{2}-\frac{1}{\rho c_{p}} \frac{\partial q_{r}}{\partial y}+\frac{Q\left(T-T_{\infty}\right)}{\rho c_{p}} \\
& +\frac{\alpha_{1}}{\rho c_{p}} \frac{\partial u}{\partial y}\left[\frac{\partial}{\partial y}\left(u \frac{\partial u}{\partial x}+v \frac{\partial u}{\partial y}\right)\right] .
\end{aligned}
$$

where, the power-law heat flux on the wall is considered in the form

$$
\begin{aligned}
& u_{w}(x)=b x^{n}, v=-v_{0}, \\
& q_{w}=-k\left(\frac{\partial T}{\partial y}\right)_{w}=D x^{s} \text { at } y=0, \\
& u \rightarrow 0, \frac{\partial u}{\partial y} \rightarrow 0, T \rightarrow T_{\infty} \text { as } \eta \rightarrow \infty,
\end{aligned}
$$

where $(x, y)$ denotes the Cartesian coordinates along the sheet and normal to it, $u$ and $v$ are the velocity components of the fluid in the $x$ - and $y$-directions, respectively, $b$ and $n$ are parameters related to the surface stretching speed, $v$ is the kinematic viscosity, $\alpha$ is the thermal diffusivity, $c_{p}$ is the specific heat at constant pressure, $q_{r}$ is the radiative heat flux and $Q$ the volumetric rate of heat generation/absorption. The radiative heat flux term by using the Rosseland approximation is given by [27]

$$
q_{r}=-\frac{4 \sigma^{*}}{3 k^{*}} \frac{\partial T^{4}}{\partial y},
$$


where $\sigma^{*}$ and $k^{*}$ are the Stefan-Boltzmann constant and the mean absorption coefficient, respectively. We can consider that the temperature differences within the flux are sufficiently small such that the term $T^{4}$ may be expressed as a linear function of temperature by expanding $T^{4}$ in a Taylor series about $T_{\infty}$ and neglecting higher-order terms we get [28]

$$
T^{4} \cong T_{\infty}^{4}+\left(T-T_{\infty}\right) \cdot 4 T_{\infty}^{3}=4 T_{\infty}^{3} T-3 T_{\infty}^{4} .
$$

using Equations (6) and (7), Equation (3) reduces to

$$
\begin{aligned}
& u \frac{\partial T}{\partial x}+v \frac{\partial T}{\partial y} \\
= & \left(\alpha+\frac{16 \sigma^{*} T_{\infty}^{3}}{3 \rho c_{p} k^{*}}\right) \frac{\partial^{2} T}{\partial y^{2}}+\frac{v}{c_{p}}\left(\frac{\partial u}{\partial y}\right)^{2} \\
& +\frac{Q\left(T-T_{\infty}\right)}{\rho c_{p}}+\frac{\alpha_{1}}{\rho c_{p}} \frac{\partial u}{\partial y}\left[\frac{\partial}{\partial y}\left(u \frac{\partial u}{\partial x}+v \frac{\partial u}{\partial y}\right)\right],
\end{aligned}
$$

where it can be seen that the effect of radiation is to enhance the thermal diffusivity.

Defining the following dimensionless function $u, v$ and $g$, which related to the similarity variable $\eta$ as

$$
\begin{aligned}
& g(\eta)=\frac{T-T_{\infty}}{\frac{D}{s} x^{s} \sqrt{\frac{2 v}{b(n+1)} x^{\frac{1-n}{2}}},} \\
& v=-\sqrt{\frac{b v(n+1)}{2} x^{\frac{n-1}{2}}\left[f+\frac{n-1}{n+1} \eta f^{\prime}\right],}
\end{aligned}
$$

where, $\psi$ is the free stream function that satisfies Equation (1) with

$$
u=\frac{\partial \psi}{\partial y}, v=-\frac{\partial \psi}{\partial x},
$$

Then we have the transformed momentum and energy equations together with the boundary conditions given by Equations (5) and (11) in the form

$$
\begin{aligned}
& \frac{2 n}{n+1} f^{\prime 2}-f f^{\prime \prime}-f^{\prime \prime \prime}+\frac{2 M}{n+1} f^{\prime} \\
& +\lambda\left(\frac{3 n-1}{2}\left(f^{\prime \prime 2}-2 f^{\prime \prime \prime \prime}\right)+\frac{n+1}{2} f f^{(i v)}\right)=0 \\
& g^{\prime \prime}+\left(\frac{3 N_{R}}{3 N_{R}+4}\right) \sigma g^{\prime} f-\frac{3 n N_{R}}{\left(3 N_{R}+4\right)(n+1)}(2 s+1-n) \sigma g f^{\prime} \\
& +\left(\frac{2}{n+1}\right)\left(\frac{3 N_{R}}{3 N_{R}+4}\right) \sigma N_{\beta} g \\
& +\left(\frac{3 N_{R}}{3 N_{R}+4}\right) \sigma E_{c} x \frac{5 n-1-2 s}{2} \\
& \cdot\left(f^{\prime \prime 2}+\lambda f^{\prime \prime}\left(\frac{3 n-1}{2} f^{\prime \prime \prime}-\frac{n+1}{2} f f^{\prime \prime \prime}\right)\right)=0
\end{aligned}
$$

$$
\begin{aligned}
& \text { If } s=\frac{5 n-1}{2}, \text { we find from (13) that } \\
& g^{\prime \prime}+\left(\frac{3 N_{R}}{3 N_{R}+4}\right) \sigma g^{\prime} f-\left(\frac{3 N_{R}}{3 N_{R}+4}\right)\left(\frac{4 n}{n+1}\right) \sigma g f^{\prime} \\
& +\left(\frac{2}{n+1}\right)\left(\frac{3 N_{R}}{3 N_{R}+4}\right) N_{\beta} \sigma g \\
& +\left(\frac{3 N_{R}}{3 N_{R}+4}\right) \sigma E_{c}\left(f^{\prime \prime 2}+\lambda f\left(\frac{3 n-1}{2} f^{\prime \prime \prime}-\frac{n+1}{2} f f^{\prime \prime \prime}\right)\right) \\
& =0
\end{aligned}
$$

The transformed boundary conditions are

$$
\left.\begin{array}{l}
f=R, f^{\prime}=1, g^{\prime}(0)=-1 \text { at } \eta=0 \\
f^{\prime} \rightarrow 0, f^{\prime \prime} \rightarrow 0, g(\infty)=0 \text { as } \eta \rightarrow \infty
\end{array}\right\}
$$

Here the prime denotes differentiation with respect to the independent similarity variable $\eta$. Moreover,

$\lambda=\frac{\alpha_{1} b x^{n-1}}{\rho v}$ is the viscoelastic parameter, $M=\frac{\sigma^{0} B_{0}^{2}}{\rho b x^{n-1}}$ is the magnetic parameter, $R=v_{0} \sqrt{\frac{2}{(n+1) v b x^{n-1}}}$ is the suction parameter, $\sigma=\frac{v}{\alpha}$ is the Prandtl number, $E_{c}=\frac{k b^{2}}{D c_{p}} \sqrt{\frac{b(n+1)}{2 v}}$ is the Eckert number, $N_{\beta}=\frac{Q}{\rho c_{p} b x^{n-1}}$ is the heat source/sink parameter, $N_{R}=\frac{k k^{*}}{4 \sigma^{*} T_{\infty}^{3}} \quad$ is the radiation parameter and $k_{0}=\frac{3 N_{R}}{3 N_{R}+4} \quad$ (with thermal radiation); $k_{0}=1$ (without thermal radiation).

The shear stress at the stretched surface is defined as

$$
\tau_{w}=\mu\left(\frac{\partial u}{\partial y}\right)_{w},
$$

using Equations (9) and (10) we have,

$$
\tau_{w}=\mu b \sqrt{\frac{b(n+1)}{2 v}} x^{\frac{3 n-1}{2}} f^{\prime \prime}(0)
$$

\section{Results and Discussion}

A boundary layer problem for momentum and heat transfer in a viscoelastic fluid flow over a non-isothermal porous stretching sheet, in the presence of thermal radiation, is examined in this paper. Stretching of the porous boundary, viscous dissipation, temperature dependent heat source/sink and thermal radiation are taken into consid- 
eration in this study. The basic boundary layer partial differential equations, which are highly non-linear, have been converted into a set of nonlinear ordinary differential equations by applying suitable similarity transformations and their numerical solutions are obtained with a finite difference code which implements the three-stage Lobatto IIIa formula is used to solve that system [29-31]. In order to verify the accuracy of the present numerical method, the results are compared with those reported earlier by [26] for the case of linear stretching sheet. The results of these comparisons are shown in Table 1. It can be seen from this table that excellent agreement between the results exists. This lends confidence to the numerical results.

The effects of viscous dissipation, work due to deformation, internal heat generation/absorption and thermal radiation are considered in the energy equation and the variations of dimensionless surface temperature, dimensionless velocity profiles as well as the heat transfer characteristics with various values of non-dimensional viscoelastic parameter $\lambda$, nonlinear stretching parameter $n$, heat source/sink parameter $N_{\beta}$, magnetic parameter $M$, suction parameter $R$, Prandtl number $\sigma$, Eckert number $E_{c}$ and radiation parameter $N_{R}$ shown in Figures 1-10.

Also, the values of wall temperature $g(0)$ for various values of physical parameters are shown in Tables 2 and 3 with and without taking the work done by deformation at the energy equation, respectively. There are many results which can be obtained from those tables. For more details, Increasing the heat source parameter $N_{\beta}$ or, the Eckert number $E_{c}$, or the magnetic parameter $M$, or the radiation parameter $N_{R}$ leads to an increasing in the wall temperature $g(0)$ when the work done by defor- mation is taken in account and this is also true for the second case, where $\sigma, R, n$ and $\lambda$ have an opposite behavior.

The plots in Figures 1(a) and (b) show the temperature distribution $g(\eta)$ for different values of the heat source/sink parameter with two cases of the radiation; in the case of existence of the thermal radiation and absence of the thermal radiation with and without work done by deformation. It is obvious that, the effect of increasing the strength of the heat sink is to increase the temperature profile, and the opposite behavior is seen for a heat source. In contrast of thermal radiation, the existence the work done by deformation is to decrease the temperature profile.

From Figures 2(a) and (b), we can see that the effect of increasing values of Prandtl number $\sigma$ is to decrease temperature at a point in the flow field, as there would be a thinning of the thermal boundary layer as a result of reduced thermal conductivity. On the other hand Figures 3(a) and (b), demonstrate the effect of the non linear parameter $n$ and it is evident that, the temperature profile decreases with increasing the values of the non linear stretching parameter. Moreover, we can see the effect of increasing the Eckert number $E_{c}$ from Figures 4(a) and (b) for the same cases disused in Figures 1(a) and (b), which is to increase the temperature distribution.

Figures 5(a) and (b) depict the effect of the magnetic field $M$, by analyzing these graphs, we see that the effect of increasing values of $M$ is to increase the temperature distribution in the boundary layer. This is because of the fact that the introduction of transverse magnetic field to an electrically conducting fluid gives rise to a resistive force, known as Lorentz force. This force has a tendency to slow down the motion of the fluid in the

Table 1. Comparison results of $g(0)$ for various values of $\sigma, N_{R}$ and $E_{c}$ with $n=0.0$ (Nonlinear stretching sheet).

\begin{tabular}{|c|c|c|c|c|c|c|}
\hline \multirow{2}{*}{$\lambda$} & \multirow{2}{*}{$E_{c}$} & \multirow{2}{*}{$N_{R}$} & \multirow{2}{*}{$\sigma$} & \multirow{2}{*}{$N_{\beta}$} & \multicolumn{2}{|c|}{$g(0)$} \\
\hline & & & & & Cortell [26] & Present Result \\
\hline 0.2 & 0.02 & 1.0 & 3.0 & 0.05 & 0.671732 & 0.6548280 \\
\hline 0.5 & & & & & 0.651127 & 0.6421121 \\
\hline \multirow[t]{11}{*}{0.2} & 0.0 & & & & 0.646621 & 0.6494326 \\
\hline & 0.4 & & & & 0.757167 & 0.7573403 \\
\hline & 0.02 & 5.0 & & & 0.462286 & 0.4647484 \\
\hline & & 8.0 & & & 0.441677 & 0.4443301 \\
\hline & & 1.0 & 1.0 & & 1.291859 & 1.2864327 \\
\hline & & & 7.0 & & 0.406628 & 0.4087103 \\
\hline & & & 3.0 & -0.25 & 0.597791 & 0.5987765 \\
\hline & & & & -0.15 & 0.614045 & 0.6153823 \\
\hline & & & & 0.0 & 0.641036 & 0.6440087 \\
\hline & & & & 0.15 & 0.693218 & 0.6790583 \\
\hline & & & & 0.25 & 0.716127 & 0.7079965 \\
\hline
\end{tabular}


Table 2. Wall temperature $g(0)$ with work done by deformation.

\begin{tabular}{|c|c|c|c|c|c|c|c|c|}
\hline \multirow{2}{*}{$N_{\beta}$} & \multirow{2}{*}{$\sigma$} & \multirow{2}{*}{$n$} & \multirow{2}{*}{$E_{c}$} & \multirow{2}{*}{$M$} & \multirow{2}{*}{$R$} & \multirow{2}{*}{$\lambda$} & \multicolumn{2}{|c|}{$g(0)$} \\
\hline & & & & & & & With Radiation & Without Radiation \\
\hline-0.2 & 8.0 & $1 / 3$ & 0.02 & 1.0 & 0.1 & 0.15 & 0.4259812 & 0.2648705 \\
\hline 0.0 & & & & & & & 0.4802939 & 0.2925684 \\
\hline 0.2 & & & & & & & 0.5710018 & 0.3321801 \\
\hline \multirow[t]{20}{*}{-0.2} & 4.0 & & & & & & 0.6397695 & 0.3900380 \\
\hline & 7.0 & & & & & & 0.4600843 & 0.2850470 \\
\hline & 10.0 & & & & & & 0.3750452 & 0.2345641 \\
\hline & 8.0 & 1.0 & & & & & 0.3496030 & 0.2190434 \\
\hline & & 3.0 & & & & & 0.2987571 & 0.1891062 \\
\hline & & 5.0 & & & & & 0.2848280 & 0.1811730 \\
\hline & & $1 / 3$ & 0.0 & & & & 0.4116075 & 0.2478815 \\
\hline & & & 0.05 & & & & 0.4475416 & 0.2903539 \\
\hline & & & 0.1 & & & & 0.4834757 & 0.3328264 \\
\hline & & & 0.02 & 0.5 & & & 0.4139565 & 0.2572655 \\
\hline & & & & 0.8 & & & 0.4214058 & 0.2619547 \\
\hline & & & & 1.5 & & & 0.4364144 & 0.2716140 \\
\hline & & & & 1.0 & 0.05 & & 0.4438452 & 0.2808115 \\
\hline & & & & & 0.5 & & 0.3132555 & 0.1747897 \\
\hline & & & & & 1.0 & & 0.2270719 & 0.1181463 \\
\hline & & & & & 1.5 & & 0.1753241 & 0.0886570 \\
\hline & & & & & 0.1 & 0.05 & 0.4271187 & 0.2653735 \\
\hline & & & & & & 0.1 & 0.4265433 & 0.2651201 \\
\hline & & & & & & 0.3 & 0.4243760 & 0.2641472 \\
\hline & & & & & & 0.7 & 0.4206671 & 0.2624275 \\
\hline
\end{tabular}

Table 3. Wall temperature $g(0)$ with work done by deformation.

\begin{tabular}{|c|c|c|c|c|c|c|c|c|}
\hline \multirow{2}{*}{$N_{\beta}$} & \multirow{2}{*}{$\sigma$} & \multirow{2}{*}{$n$} & \multirow{2}{*}{$E_{c}$} & \multirow{2}{*}{$M$} & \multirow{2}{*}{$R$} & \multirow{2}{*}{$\lambda$} & \multicolumn{2}{|c|}{$g(0)$} \\
\hline & & & & & & & With Radiation & Without Radiation \\
\hline-0.2 & 8.0 & $1 / 3$ & 0.02 & 1.0 & 0.1 & 0.15 & 0.5083353 & 0.2642551 \\
\hline 0.0 & & & & & & & 0.5798098 & 0.2918004 \\
\hline 0.2 & & & & & & & 0.7017604 & 0.3311588 \\
\hline \multirow[t]{20}{*}{-0.2} & 4.0 & & & & & & 0.7102198 & 0.3894706 \\
\hline & 7.0 & & & & & & 0.5400828 & 0.2844400 \\
\hline & 10.0 & & & & & & 0.4613835 & 0.2339356 \\
\hline & 8.0 & 1.0 & & & & & 0.4018430 & 0.2172851 \\
\hline & & 3.0 & & & & & 0.3274262 & 0.1856562 \\
\hline & & 5.0 & & & & & 0.3050635 & 0.1767095 \\
\hline & & $1 / 3$ & 0.0 & & & & 0.4116075 & 0.2478815 \\
\hline & & & 0.05 & & & & 0.6534269 & 0.2888155 \\
\hline & & & 0.1 & & & & 0.8952464 & 0.3297495 \\
\hline & & & 0.02 & 0.5 & & & 0.4755726 & 0.2568651 \\
\hline & & & & 0.8 & & & 0.4957535 & 0.2614253 \\
\hline & & & & 1.5 & & & 0.5374969 & 0.2707835 \\
\hline & & & & 1.0 & 0.05 & & 0.5272657 & 0.2803169 \\
\hline & & & & & 0.5 & & 0.3862716 & 0.1732892 \\
\hline & & & & & 1.0 & & 0.2889979 & 0.1157326 \\
\hline & & & & & 1.5 & & 0.2281186 & 0.0854866 \\
\hline & & & & & 0.1 & 0.05 & 0.5126295 & 0.2651608 \\
\hline & & & & & & 0.1 & 0.5023476 & 0.2629805 \\
\hline & & & & & & 0.3 & 0.4888003 & 0.2600536 \\
\hline & & & & & & 0.7 & 0.5083353 & 0.2642551 \\
\hline
\end{tabular}



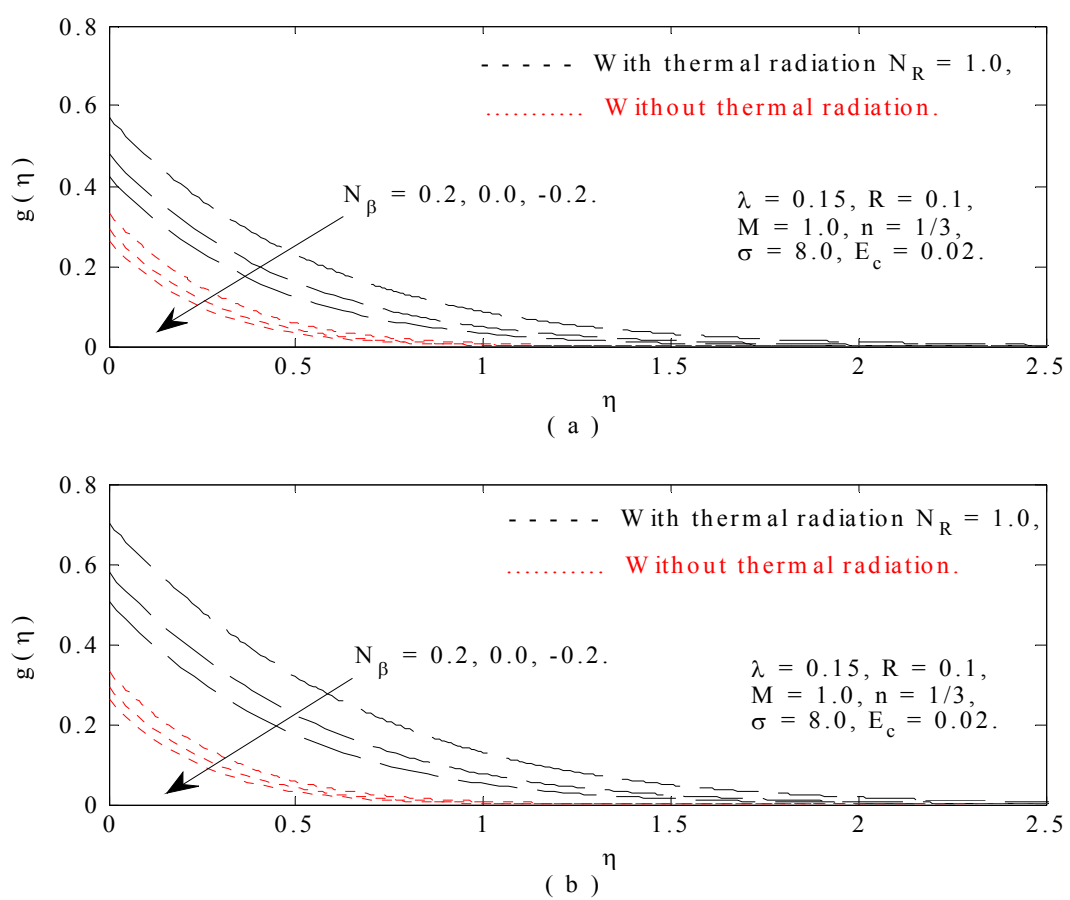

Figure 1. Temperature profiles for several values of $N_{\beta}$ (a) with and (b) without work done by deformation.
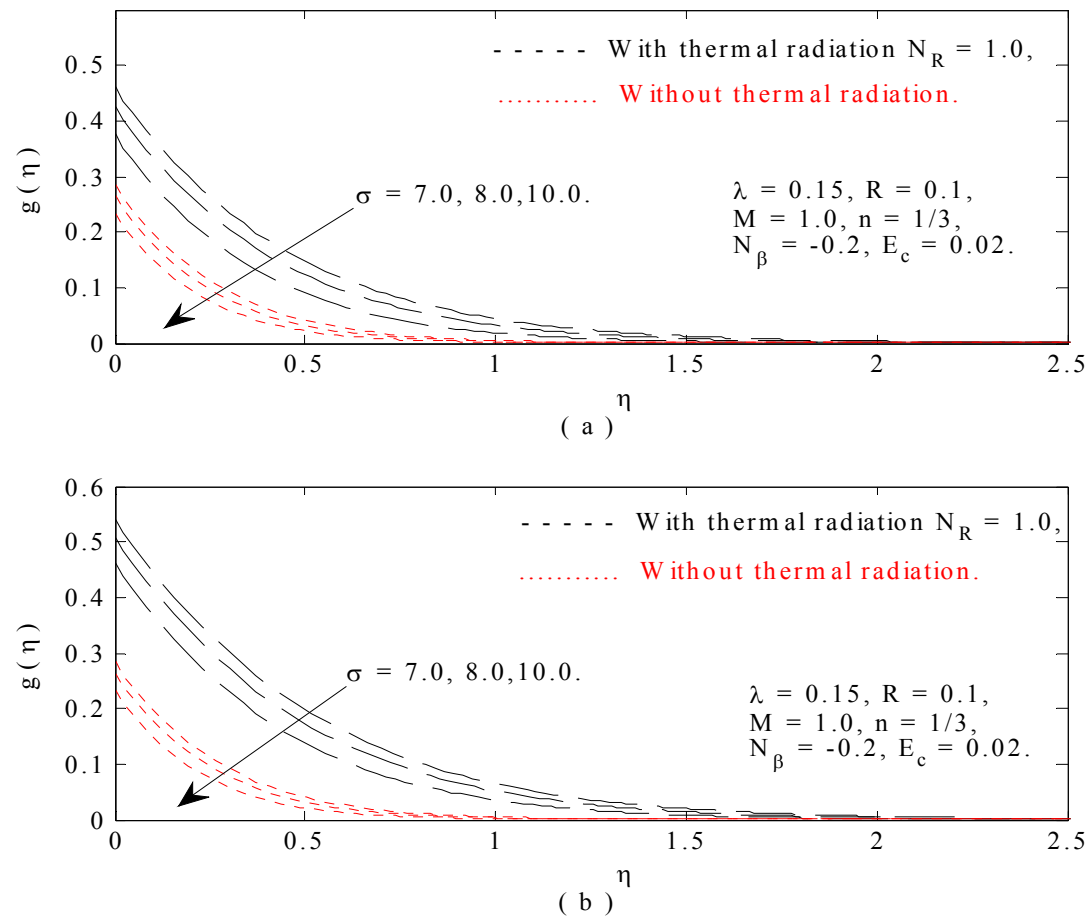

Figure 2. Temperature profiles for several values of $\sigma(a)$ with and (b) without work done by deformation.

boundary layer and to increase the temperature distribution, where the effect of the suction parameter is plotted in Figures 6(a) and (b) and the effect of the viscoelastic parameter $\lambda$ is clearly shown in Figures 7(a) and (b) with all cases.

Velocity profiles with $n=0.5,3.0$ for various values of $M$ is shown in Figure 8(a) where the same values is plotted in Figure 8(b) but for $R=1.0$. Also, the same values of Figures 8(a) and (b) are Plotted for different values of the viscoelastic parameter in Figures 9(a) and (b). We can see from those figures that, a faster motion is considered when the viscoelastic parameter increases 

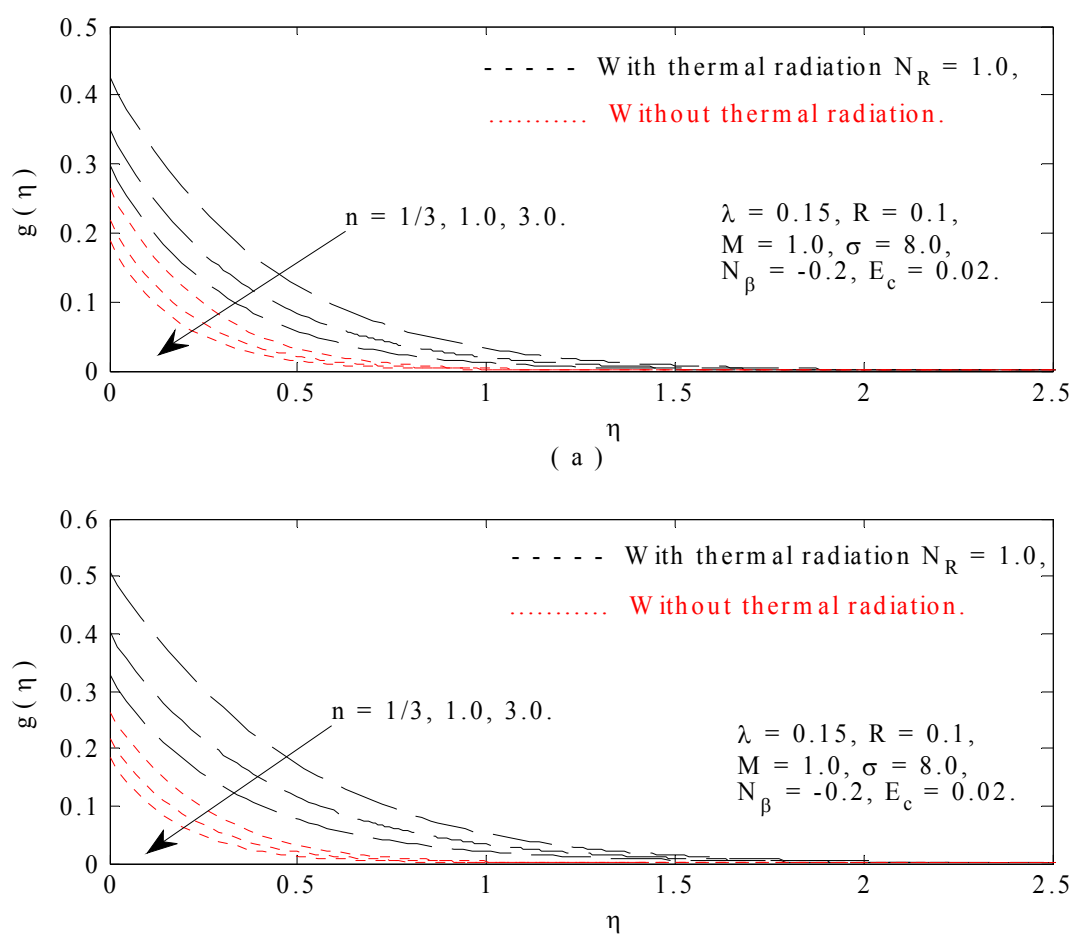

$(\mathrm{b})^{\eta}$

Figure 3. Temperature profiles for several values of $\boldsymbol{n}$ (a) with and (b) without work done by deformation.
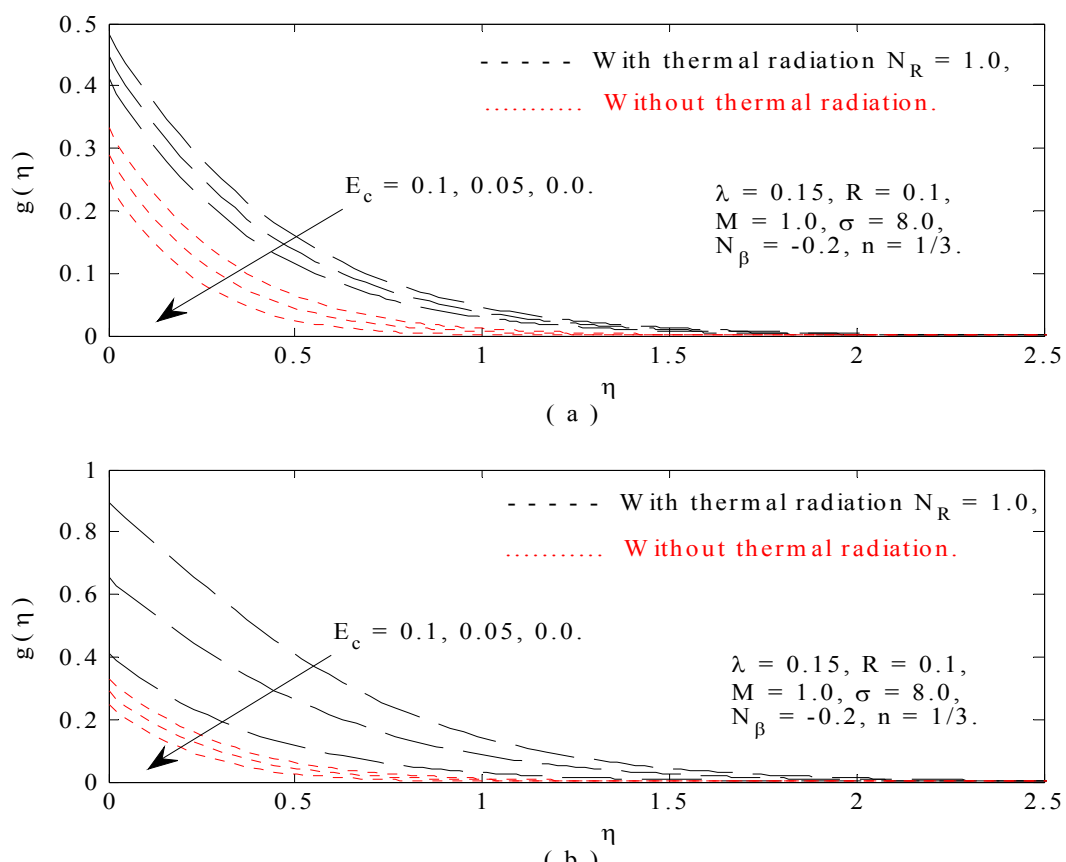

Figure 4. Temperature profiles for several values of $E_{c}$ (a) with and (b) without work done by deformation.

whereas it is slower when the suction parameter and magnetic parameter increase. Finally, Figure 10 shows variation of skin fraction coefficient against $R$ for different values of $M, n, \lambda$, from which we can say that, with an increasing in the nonlinear stretching parameter $n$ or the viscoelastic parameter $\lambda$ tends to a decreasing in the wall shear stress which represented in terms of $-f^{\prime \prime}(0)$ as defined by Equation (17) i.e. the velocity profile increases, but the opposite effect is seen for the magnetic parameter $M$. 

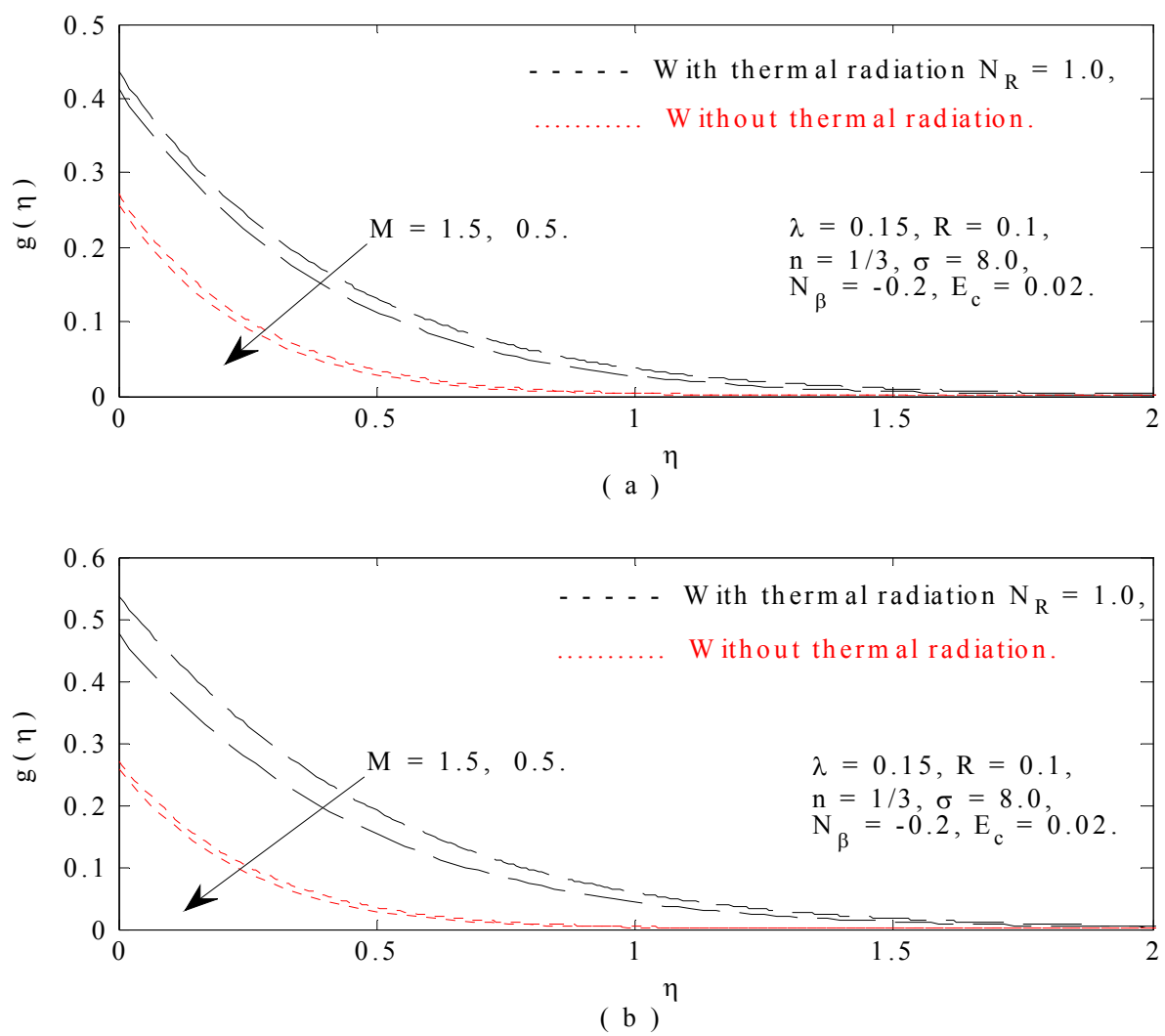

Figure 5. Temperature profiles for several values of $M($ a) with and (b) without work done by deformation.
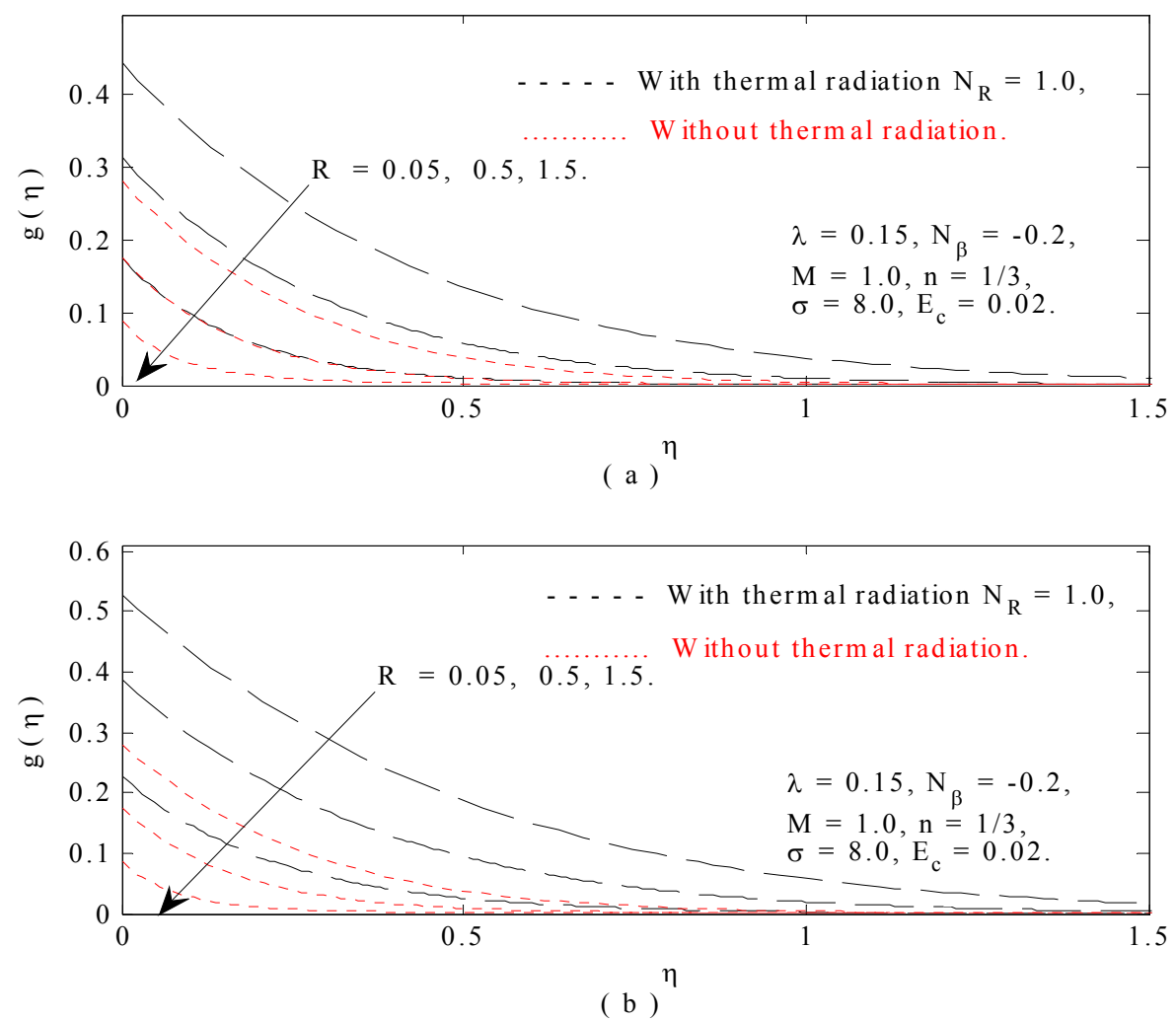

Figure 6. Temperature profiles for several values of $\boldsymbol{R}$ (a) with and (b) without work done by deformation. 

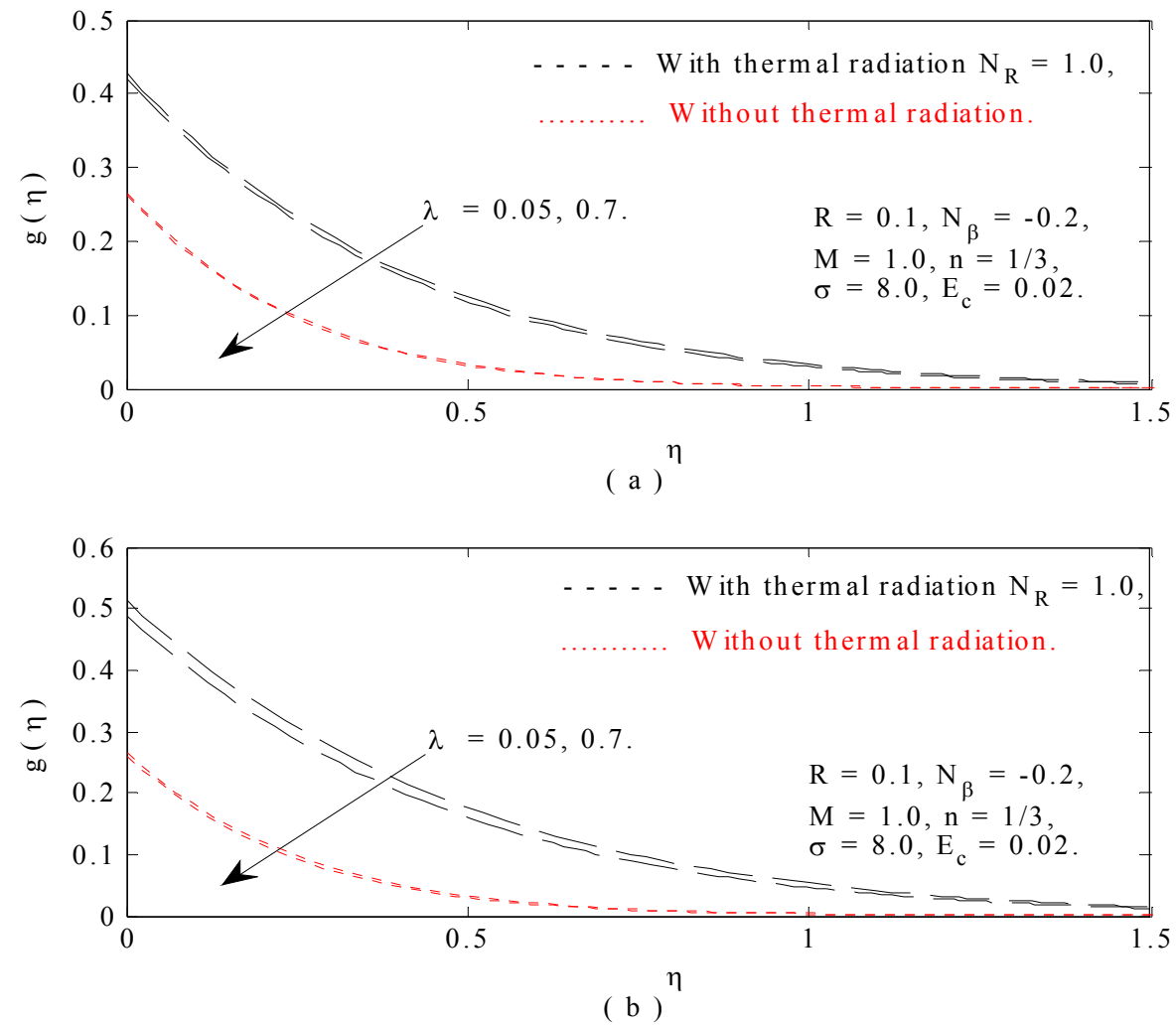

Figure 7. Temperature profiles for several values of $\lambda$ (a) with and (b) without work done by deformation.

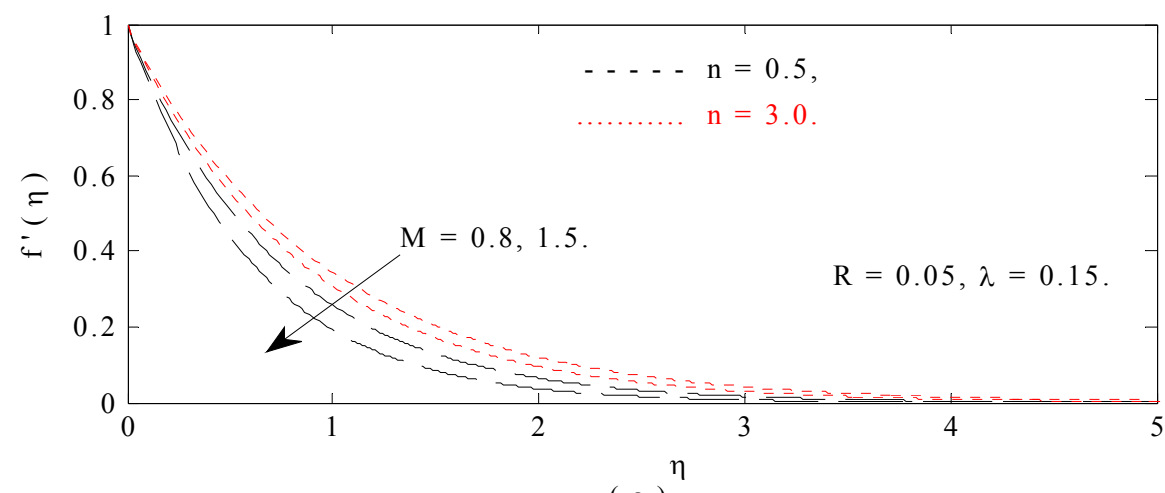

( a )

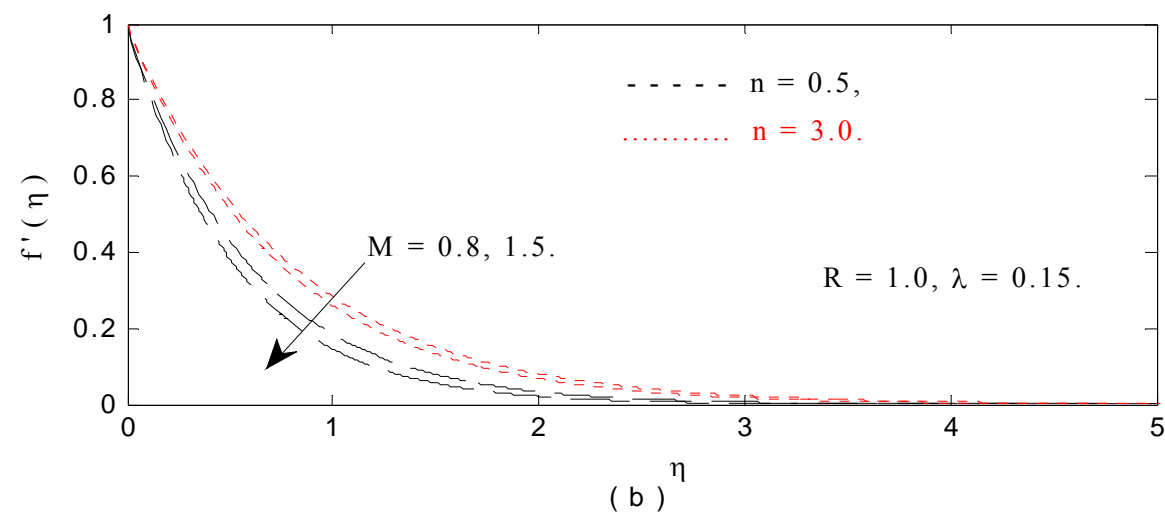

Figure 8. Velocity profiles with $n=0.5,3.0$ for various values of $M$ and $R$. 

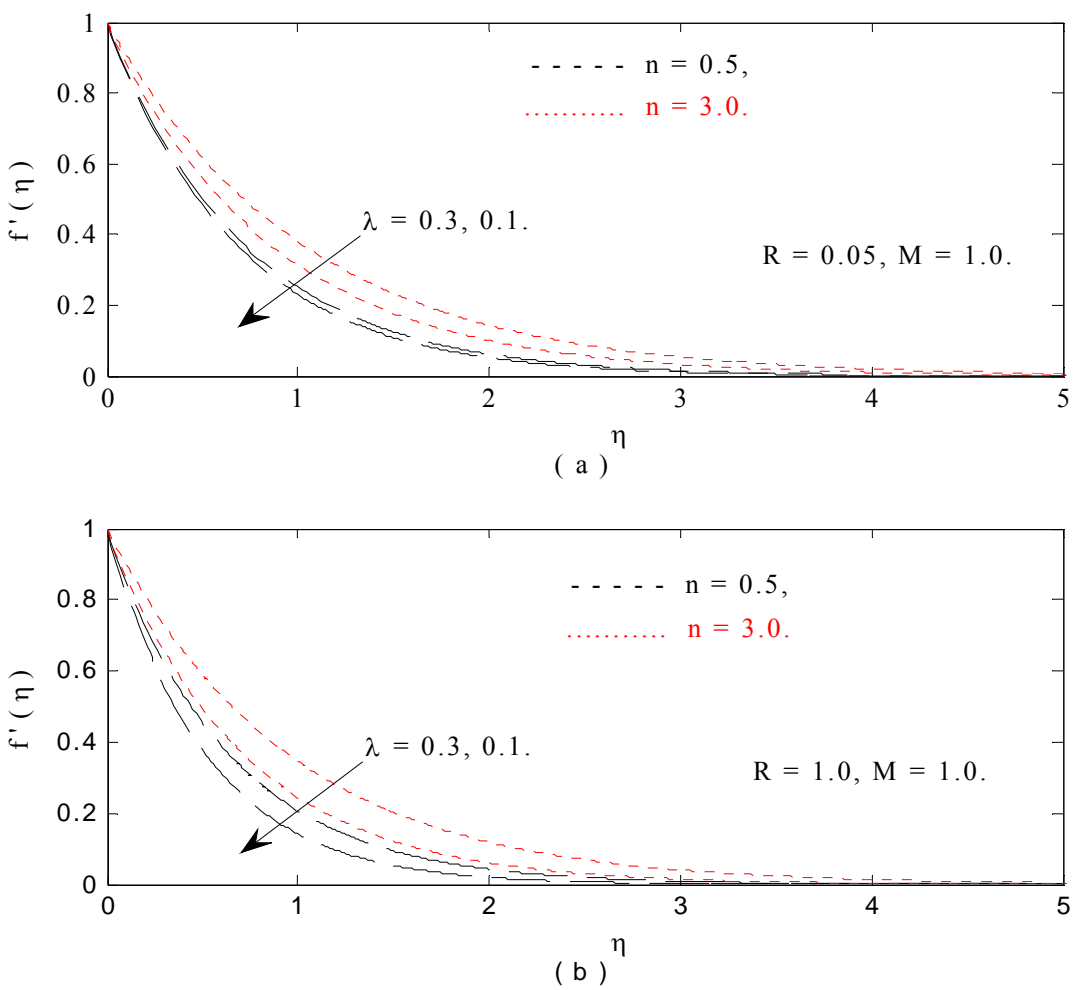

Figure 9. Velocity profiles with $n=0.5,3.0$ for various values of $\lambda$ and $R$.

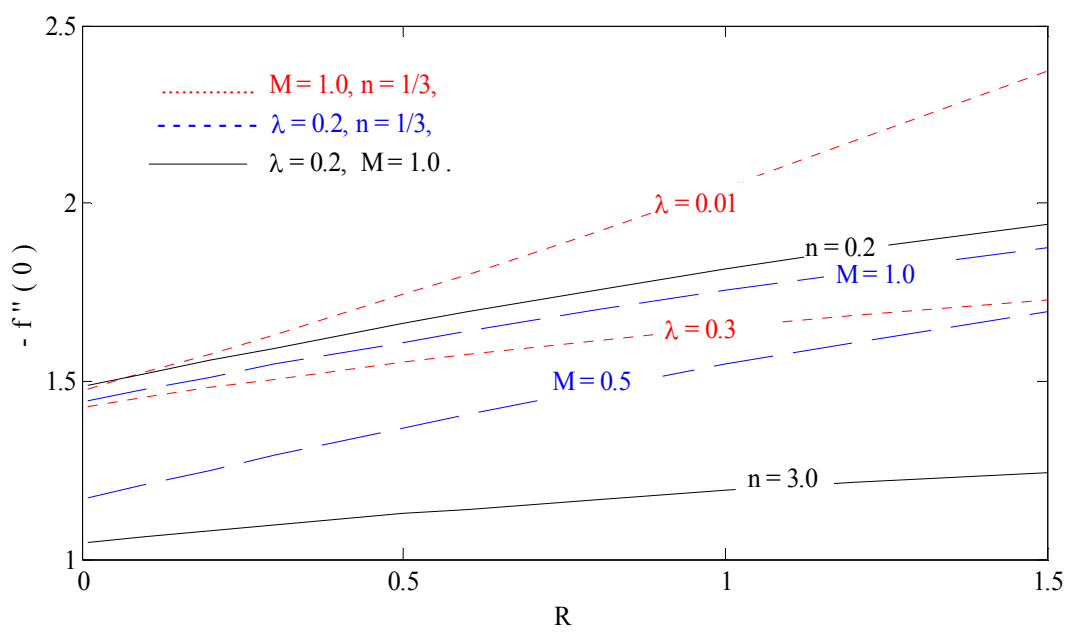

Figure 10. Variation of skin fraction coefficient against $R$ for different values of $M, n$, and $\lambda$.

\section{Conclusions}

The effects of heat source/sink, radiation and work done by deformation on flow and heat transfer of a viscoelastic fluid over a nonlinear stretching sheet have been investigated. Numerical solutions for momentum and heat transfer are obtained. In the light of the numerical results the following conclusions may be drawn:

- A faster motion is considered when the viscoelastic parameter or the nonlinear stretching parameter increases whereas it is slower when the suction pa- rameter and the magnetic force increase.

- The effect of increasing values of magnetic parameter is to increase the temperature distribution in the boundary layer and so does the Eckert number.

- The influence of the work due to deformation has significance effect of the temperature distribution.

- The presence of the thermal radiation term in the energy equation yields an augment in the fluid's temperature.

- The internal heat generation/absorption enhances or damps the heat transport. Finally, increasing suction 
parameter or viscoelastic parameter or Prandtl number is to decrease the temperature distribution in the flow region.

\section{REFERENCES}

[1] A. C. Truesdell and W. Noll, "The Non-Linear Field Theories of Mechanics," In: S. Flugge, Ed., Encyclopedia of Physics, Springer, Berlin, 1965, pp. 1-591.

[2] K. R. Rajagopal, "On Boundary Conditions for Fluids of the Differential Type," In: A. Sequeira, Ed., NavierStokes Equations and Related Non-linear Problems, Plenum Press, New York, 1995, pp. 273-278.

[3] K. R. Rajagopal and P. N. Kaloni, "Some Remarks on Boundary Conditions for Fluids of the Differential Type," In: G. A. C. Graham and S. K. Malik, Eds., Continuum Mechanics and Its Applications, Hemisphere, New York, 1989, pp. 935-942.

[4] K. R. Rajagopal and A. S. Gupta, "An Exact Solution for the Flow of a Non-Newtonian Fluid past an Infinite Plate," Meccanica, Vol. 19, No. 2, 1984, pp. 158-160. doi:10.1007/BF01560464

[5] D. W. Beard and K. Walters, "Elastico-Viscous Boundary Layer Flows," Proceedings of the Cambridge Philosophical Society, Vol. 60, 1964, pp. 667-674. doi: $10.1017 / \mathrm{S} 0305004100038147$

[6] J. E. Danberg and K. S. Fansler, "A Non Similar Moving Wall Boundary-Layer Problem," Quarterly of Applied Mathematics, Vol. 34, 1976, pp. 305-309.

[7] K. R. Rajagopal, T. Y. Na and A. S. Gupta, "Flow of a Viscoelastic Fluid over a Stretching Sheet," Rheologica Acta, Vol. 23, No. 2, 1984, pp. 213-215. doi:10.1007/BF01332078

[8] B. S. Dandapat and A. S. Gupta, "Flow and Heat Transfer in a Viscoelastic Fluid over a Stretching Sheet," International Journal of Non-Linear Mechanics, Vol. 24, No. 3, 1989, pp. 215-219. doi:10.1016/0020-7462(89)90040-1

[9] F. M. Hady and R. S. R. Gorla, "Heat Transfer from a Continuous Surface in a Parallel Free Stream of Viscoelastic Fluid," Acta Mechanica, Vol. 128, No. 3, 1998, pp. 201-208. doi:10.1007/BF01251890

[10] C. I. Cookey, A. Ogulu and V. B. Omubo-Pepple, "Influence of Viscous Dissipation and Radiation on Unsteady MHD Free-Convection Flow past an Infinite Heated Vertical Plate in a Porous Medium with Time-Dependent Suction," International Journal of Heat and Mass Transfer, Vol. 46, No. 13, 2003, pp. 2305-2311. doi:10.1016/S0017-9310(02)00544-6

[11] M. Kumari and G. Nath, "Radiation Effect on Mixed Convection from a Horizontal Surface in a Porous Medium," Mechanics Research Communications, Vol. 31, No. 4, 2004, pp. 483-491. doi:10.1016/j.mechrescom.2003.11.006

[12] M. A. Abd El-Naby, E. M. E. Elbarbary and N. Y. Abdelazem, "Finite Difference Solution of Radiation Effects on MHD Unsteady Free-Convection Flow over Vertical Porous Plate," Applied Mathematics and Computation, Vol. 151, No. 2, 2004, pp. 327-346.
doi:10.1016/S0096-3003(03)00344-8

[13] S. Abel, K. V. Prasad and A. Mahaboob, "Bouyancy Force and Thermal Radiation Effects in MHD BoundaryLayer Viscoelastic Fluid Flow over Continuously Moving Stretching Surface," International Journal of Thermal Sciences, Vol. 44, No. 5, 2005, pp. 465-476. doi:10.1016/j.ijthermalsci.2004.08.005

[14] S. K. Khan, "Heat Transfer in a Viscoelastic Fluid Flow over a Stretching Surface with Heat Source/Sink, Suction/ Blowing and Radiation," International Journal of Heat and Mass Transfer, Vol. 49, No. 3-4, 2006, pp. 628-639. doi:10.1016/j.ijheatmasstransfer.2005.07.049

[15] Mahdy and H. M. ElShehabey, "Uncertainties in Physical Property Effects on Viscous Flow and Heat Transfer over a Nonlinearly Stretching Sheet with Nanofluids," International Journal of Heat and Mass Transfer, Vol. 39, No. 5, 2012, pp. 713-719. doi:10.1016/j.icheatmasstransfer.2012.03.019

[16] R. Cortell, "Effects of Viscous Dissipation and Radiation on the Thermal Boundary Layer over a Nonlinearly Stretching Sheet," Physics Letters A, Vol. 372, No. 13, 2008, pp. 631-636. doi:10.1016/j.physleta.2007.08.005

[17] K. Vajravelu, "Viscous Flow over a Nonlinearly Stretching Sheet," Applied Mathematics and Computation, Vol. 124, No. 3, 2001, pp. 281-288. doi:10.1016/S0096-3003(00)00062-X

[18] R. Cortell, "Viscous Flow and Heat Transfer over a Nonlinearly Stretching Sheet," Applied Mathematics and Computation, Vol. 184, No. 2, 2007, pp. 864-873. doi:10.1016/j.amc.2006.06.077

[19] S. Awang Kechil and I Hashim, "Series Solution of Flow over Nonlinearly Stretching Sheet with Chemical Reaction and Magnetic Field," Physics Letters A, Vol. 372, No. 13, 2008, pp. 2258-2263. doi:10.1016/j.physleta.2007.11.027

[20] Z. Ziabakhsh, G. Domairry, H. Bararnia and H. Babazadeh, "Analytical Solution of Flow and Diffusion of Chemically Reactive Species over a Nonlinearly Stretching Sheet Immersed in a Porous Medium," Journal of the Taiwan Institute of Chemical Engineers, Vol. 41, No. 1, 2010, pp. 22-28. doi:10.1016/j.jtice.2009.04.011

[21] Muhaimina, R. Kandasamya and I. Hashimb, "Effect of Chemical Reaction, Heat and Mass Transfer on Nonlinear Boundary Layer past a Porous Shrinking Sheet in the Presence of Suction," Nuclear Engineering and Design, Vol. 240, No. 5, 2010, pp. 933-939. doi:10.1016/j.nucengdes.2009.12.024

[22] R. Gorder, "High-Order Nonlinear Boundary Value Problems Admitting Multiple Exact Solutions with Application to the Fluid Flow over a Sheet," Applied Mathematics and Computation, Vol. 216, No. 7, 2010, pp. 2177 2182. doi:10.1016/j.amc.2010.03.053

[23] R. Cortell, "Heat and Fluid Flow Due to Non-Linearly Stretching Surfaces," Applied Mathematics and Computation, Vol. 271, 2011, pp. 7564-7564. doi:10.1016/j.amc.2011.02.029

[24] R. Gorder, K. Vajravelu and F. T. Akyildiz, "Existence and Uniqueness Results for a Nonlinear Differential 
Equation Arising in Viscous Flow over a Nonlinearly Stretching Sheet," Applied Mathematics Letters, Vol. 24, No. 2, 2011, pp. 238-242. doi:10.1016/j.aml.2010.09.011

[25] K. Vajravelu, K. V. Prasad and N. S. Prasanna, "Diffusion of a Chemically Reactive Species of a Power-Law Fluid past a Stretching Surface," Computers \& Mathematics with Applications, Vol. 62, No. 1, 2011, pp. 93108. doi:10.1016/j.camwa.2011.04.055

[26] R. Cortell, "Effects of Heat Source/Sink, Radiation and Work Done by Deformation on Flow and Heat Transfer of a Viscoelastic Fluid over a Stretching Sheet," Computers \& Mathematics with Applications, Vol. 53, No. 2, 2007 pp. 305-316. doi:10.1016/j.camwa.2006.02.041

[27] P. S. Datti, K. V. Prasad, M. Subhas Abel and A. Joshi, "MHD Viscoelastic Fluid Flow over a Non-Isothermal Stretching Sheet," International Journal of Engineering Science, Vol. 42, No. 8-9, 2004, pp. 935-946. doi:10.1016/j.ijengsci.2003.09.008

[28] M. M. Rahman and T. Sultana, "Radiative Heat Transfer Flow of Micropolar Fluid with Variable Heat Flux in a Porous Medium," Nonlinear Analysis: Modelling and Control, Vol. 13, No. 1, 2008, pp. 71-87

[29] F. M. Hady, F. S. Ibrahim, H. M. El-Hawary and A. M. AbdElhady, "Forced Convection Flow of Nanofluids past Power Law Stretching Horizontal Plates," Applied Mathematics, Vol. 3, No. 2, 2012, pp. 121-126. doi:10.4236/am.2012.32019

[30] L. F. Shampine, M. W. Reichelt and J. Kierzenka, "Solving Boundary Value Problems for Ordinary Differential Equations in MATLAB with bvp4c." http://www.mathworks.com/bvp_tutorial

[31] L. F. Shampine, I. Gladwell and S. Thompson, "Solving ODEs with MATLAB," Cambridge University Press, Cambridge, 2003. doi:10.1017/CBO9780511615542 